CARPATHIAN J. MATH.

Volume 37 (2021), No. 2,

Pages 161 - 171
Online version at https : //www. carpathian. cunbm. utcluj.ro/

Print Edition: ISSN 1584 - 2851; Online Edition: ISSN 1843 - 4401

DOI: https:/ /doi.org/10.37193/CJM.2021.02.02

Dedicated to Prof. Ioan A. Rus on the occasion of his $85^{\text {th }}$ anniversary

\title{
On a Steklov eigenvalue problem associated with the $(p, q)$-Laplacian
}

\section{LUMINIŢA BARBU ${ }^{a}$ and GHEORGHE MOROŞANU ${ }^{b, c}$}

ABSTRACT. Consider in a bounded domain $\Omega \subset \mathbb{R}^{N}, N \geq 2$, with smooth boundary $\partial \Omega$, the following eigenvalue problem

$$
\begin{aligned}
& \mathcal{A} u:=-\Delta_{p} u-\Delta_{q} u=\lambda a(x)|u|^{r-2} u \text { in } \Omega, \\
& \left(|\nabla u|^{p-2}+|\nabla u|^{q-2}\right) \frac{\partial u}{\partial \nu}=\lambda b(x)|u|^{r-2} u \text { on } \partial \Omega,
\end{aligned}
$$

where $1<r<q<p<\infty$ or $1<q<p<r<\infty$; $r \in\left(1, \frac{p(N-1)}{N-p}\right)$ if $p<N$ and $r \in(1, \infty)$ if $p \geq N$; $a \in L^{\infty}(\Omega), b \in L^{\infty}(\partial \Omega)$ are given nonnegative functions satisfying

$$
\int_{\Omega} a d x+\int_{\partial \Omega} b d \sigma>0
$$

Under these assumptions we prove that the set of all eigenvalues of the above problem is the interval $[0, \infty)$. Our result complements those previously obtained by Abreu, J. and Madeira, G., [Generalized eigenvalues of the $(p, 2)-L a p l a c i a n$ under a parametric boundary condition, Proc. Edinburgh Math. Soc., 63 (2020), No. 1, 287-303], Barbu, L. and Moroşanu, G., [Full description of the eigenvalue set of the ( $p, q)$-Laplacian with a Steklovlike boundary condition, J. Differential Equations, in press], Barbu, L. and Moroşanu, G., [Eigenvalues of the negative $(p, q)$ - Laplacian under a Steklov-like boundary condition, Complex Var. Elliptic Equations, 64 (2019), No. 4, 685-700], Fărcăşeanu, M., Mihăilescu M. and Stancu-Dumitru, D., [On the set of eigen-values of some PDEs with homogeneous Neumann boundary condition, Nonlinear Anal. Theory Methods Appl., 116 (2015), 19-25], Mihăilescu, M., [An eigenvalue problem possesing a continuous family of eigenvalues plus an isolated eigenvale, Commun. Pure Appl. Anal., 10 (2011), 701-708], Mihăilescu, M. and Moroşanu, G., [Eigenvalues of $-\triangle_{p}-\triangle_{q}$ under Neumann boundary condition, Canadian Math. Bull., 59 (2016), No. 3, 606-616].

\section{INTRODUCTION}

Let $\Omega \subset \mathbb{R}^{N}$ be a bounded domain with smooth boundary $\partial \Omega$. Consider the eigenvalue problem

$$
\left\{\begin{array}{l}
\mathcal{A} u:=-\Delta_{p} u-\Delta_{q} u=\lambda a(x)|u|^{r-2} u \text { in } \Omega, \\
\frac{\partial u}{\partial \nu_{\mathcal{A}}}:=\left(|\nabla u|^{p-2}+|\nabla u|^{q-2}\right) \frac{\partial u}{\partial \nu}=\lambda b(x)|u|^{r-2} u \text { on } \partial \Omega,
\end{array}\right.
$$

where $\nu$ is the unit outward normal to $\partial \Omega$. As usual, $\Delta_{p}$ denotes the $p$-Laplacian, i.e., $\Delta_{p} u=\operatorname{div}\left(|\nabla u|^{p-2} \nabla u\right)$.

Throughout this paper, the following hypotheses will be assumed

$$
\left(h_{p q r}\right) \quad 1<r<q<p<\infty \text { or } 1<q<p<r<\infty ; r \in\left(1, \frac{p(N-1)}{N-p}\right) \text { if } 1<p<N \text { and }
$$
$r \in(1, \infty)$ if $p \geq N$;

Received: 07.01.2021. In revised form: 16.04.2021. Accepted: 16.04.2021 2010 Mathematics Subject Classification. 35J60, 35J92, 35P30.

Key words and phrases. Eigenvalues, $(p, q)$-Laplacian, Sobolev space, Nehari manifold, variational methods. Corresponding author: Gheorghe Moroşanu; morosanu@math.ubbcluj.ro 
$\left(h_{a b}\right) \quad a \in L^{\infty}(\Omega)$ and $b \in L^{\infty}(\partial \Omega)$ are given nonnegative functions satisfying

$$
\int_{\Omega} a(x) d x+\int_{\partial \Omega} b(\sigma) d \sigma>0 .
$$

Remark 1.1. Regarding the assumption $r \in\left(1, \frac{p(N-1)}{N-p}\right)$ if $1<p<N$ and $r \in(1, \infty)$ if $p \geq N$, we point out that this is directly related to the well-known embeddings $W^{1, p}(\Omega) \hookrightarrow$ $L^{r}(\Omega)$ which hold in the cases: $(i) 1 \leq r \leq p^{*}=p N /(N-p)$, if $1<p<N ;(j) p \leq r<\infty$, if $p=N ;(k) r=\infty$, if $p>N$. Moreover, these embeddings are compact when $1 \leq r<p^{*}$ in case $(i)$, all $r$ in case $(j)$, and when reinterpreted as $W^{1, p}(\Omega) \hookrightarrow C^{1}(\bar{\Omega})$ in case $(k)$. We also have trace compact embeddings $W^{1, p}(\Omega) \hookrightarrow L^{r}(\partial \Omega)$ for all $1 \leq p \leq r<p(N-1) /(N-p)$ if $1 \leq p<N$, and similarly as before in the other ranges of $p$ (see [2], [6, Section 9.3]).

The solution $u$ of (1.1) will be sought in the space $W:=W^{1, p}(\Omega)$, so the normal derivative $\frac{\partial u}{\partial \nu_{\mathcal{A}}}$ exists in a trace sense, and the above problem is satisfied in the distribution sense. According to a Green type formula (see [7], p. 71), one can define the eigenvalues of our problems in term of weak solution as follows

Definition 1.1. $\lambda \in \mathbb{R}$ is an eigenvalue of problem (1.1) if there exists $u_{\lambda} \in W \backslash\{0\}$ such that

$$
\begin{aligned}
& \int_{\Omega}\left(\left|\nabla u_{\lambda}\right|^{p-2}+\left|\nabla u_{\lambda}\right|^{q-2}\right) \nabla u_{\lambda} \cdot \nabla w d x \\
& \quad=\lambda\left(\int_{\Omega} a\left|u_{\lambda}\right|^{q-2} u_{\lambda} w d x+\int_{\partial \Omega} b\left|u_{\lambda}\right|^{q-2} u_{\lambda} w d \sigma\right) \forall w \in W .
\end{aligned}
$$

According to the above remark, all the integral terms in Definition 1.1 make sense.

Conversely, by virtue of the same Green formula, if $\lambda$ is an eigenvalue then any eigenfunctions $u_{\lambda} \in W \backslash\{0\}$ corresponding to it satisfies problem (1.1) in the distribution sense. Our goal is to determine the set of all eigenvalues of problem (1.1).

The main result of this paper is given by the following theorem

Theorem 1.1. Assume that $\left(h_{p q r}\right)$ and $\left(h_{a b}\right)$ above are fulfilled. Then the set of eigenvalues of problem $(1.1)$ is $[0, \infty)$.

Remark 1.2. It is worth mentioning that if $b \equiv 0$ (Neumann boundary condition) and $1<p<N$, Theorem (1.1) holds if the condition $1<r<p(N-1) /(N-p)$ is replaced by the weaker condition $1<r<p N /(N-p)$.

In the case $q=r=2, a \equiv 1, b \equiv 0$, the set of eigenvalues for problem (1.1) was completely described by M. Mihăilescu [11] (for $p>2$ ) and M. Fărcăşeanu, M. Mihăilescu and D. Stancu-Dumitru [9] (for $p \in(1,2)$ ). Problem (1.1) with $q=r=2, p \in(1, \infty) \backslash$ $\{2\}$, was studied by J. Abreu and G. Madeira [1]. Note also that problem (1.1) with $p \in$ $(1, \infty), r=q \in(2, \infty), p \neq q, a \equiv 1, b \equiv 0$, was investigated by M. Mihăilescu and G. Moroşanu in [12]; also, problem (1.1) with $p, q \in(1, \infty), p \neq q, r=q$ was solved by L. Barbu and G. Moroşanu [3, 4].

\section{PRELIMINARY RESUlTS}

Choosing $w=u_{\lambda}$ in (1.3) shows that the eigenvalues of problem (1.1) cannot be negative. It is also obvious that $\lambda_{0}=0$ is an eigenvalue of this problem and the corresponding eigenfunctions are the nonzero constant functions. So any other eigenvalue belongs to $(0, \infty)$ 
If we assume that $\lambda>0$ is an eigenvalue of problem (1.1) and choose $w \equiv 1$ in (1.3) we deduce that every eigenfunction $u_{\lambda}$ corresponding to $\lambda$ satisfies the equation

$$
\int_{\Omega} a\left|u_{\lambda}\right|^{r-2} u_{\lambda} d x+\int_{\partial \Omega} b\left|u_{\lambda}\right|^{r-2} u_{\lambda} d \sigma=0 .
$$

So all eigenfunctions corresponding to positive eigenvalues necessarily belong to the set

$$
\mathcal{C}_{r}:=\left\{u \in W ; \int_{\Omega} a|u|^{r-2} u d x+\int_{\partial \Omega} b|u|^{r-2} u d \sigma=0\right\} .
$$

This set is a symmetric cone. Moreover, $\mathcal{C}_{r}$ is a weakly closed subset of $W:=W^{1, p}(\Omega)$. Indeed, let $\left(u_{n}\right)_{n} \subset \mathcal{C}_{r}$ such that $u_{n} \rightarrow u_{0}$ in $W$. Since $W \hookrightarrow L^{r}(\Omega)$ and $W \hookrightarrow L^{r}(\partial \Omega)$ compactly, there exists a subsequence of $\left(u_{n}\right)_{n^{\prime}}$ also denoted $\left(u_{n}\right)_{n}$, such that

$$
u_{n} \rightarrow u_{0} \text { in } L^{r}(\Omega), u_{n} \rightarrow u_{0} \text { in } L^{r}(\partial \Omega) .
$$

By Lebesgue's Dominated Convergence Theorem (see also [6, Theorem 4.9]) we obtain $u_{0} \in \mathcal{C}_{r}$. In addition, $\mathcal{C}_{r}$ has nonzero elements (see [4, Section 2]).

Let $\mathcal{K}_{r}: W \rightarrow \mathbb{R}$ be the $C^{1}$-functional defined by

$$
\mathcal{K}_{r}(u):=\int_{\Omega} a|u|^{r} d x+\int_{\partial \Omega} b|u|^{r} d \sigma \forall u \in W .
$$

Remark 2.3. If for some $\lambda>0, u \in W \backslash\{0\}$ satisfies the equation

$$
\int_{\Omega}\left(|\nabla u|^{p}+|\nabla u|^{q}\right) d x=\lambda \mathcal{K}_{r}(u)
$$

then $u$ cannot be a constant function (see assumption (1.2)) and so $\mathcal{K}_{r}(u)>0$. Therefore, denoting $\Gamma_{1}(u):=\{x \in \Omega ; a(x) u(x) \neq 0\}, \Gamma_{2}(u):=\{x \in \partial \Omega ; b(x) u(x) \neq 0\}$, we see that either $\left|\Gamma_{1}(u)\right|_{N}>0$ or $\left|\Gamma_{2}(u)\right|_{N-1}>0$.

Obviously $u_{\lambda}$ corresponding to any eigenvalue $\lambda>0$ cannot be a constant function (see (1.3) with $v=u_{\lambda}$ and (1.2)).

The following lemmas are useful in the proof of Theorem 1.1.

Lemma 2.1. If hypotheses $\left(h_{a b}\right)$ hold and $r \in\left(1, \frac{p(N-1)}{N-p}\right)$ for $1<p<N$ and $r \in(1, \infty)$ for $p \geq N$, then the following norm is equivalent with the usual norm (denoted by $\|\cdot\|_{W}$ ) of the Sobolev space $W=W^{1, p}(\Omega)$

$$
\|u\|_{r}:=\|\nabla u\|_{L^{p}(\Omega)}+\left(\mathcal{K}_{r}(u)\right)^{\frac{1}{r}} \forall u \in W .
$$

Proof. This fact follows from [8, Proposition 3.9.55]. Indeed, $\left(\mathcal{K}_{r}(u)\right)^{\frac{1}{r}}$ is a seminorm which satisfies the two requirements of that proposition

(j) $\exists d>0$ such that $\left(\mathcal{K}_{r}(u)\right)^{\frac{1}{r}} \leq d\|u\|_{W} \quad \forall u \in W$, and

(jj) if $u=$ constant, then $\left(\mathcal{K}_{r}(u)\right)^{\frac{1}{r}}=0$ implies $u \equiv 0$.

Lemma 2.2. If hypotheses $\left(h_{a b}\right)$ hold and $r \in\left(1, \frac{p(N-1)}{N-p}\right)$ for $1<p<N$ and $r \in(1, \infty)$ for $p \geq N$, then there exists a positive constant $C$ which depends on $p, r, N$ and $\Omega$, such that for every $u \in \mathcal{C}_{r}$

$$
\left(\mathcal{K}_{r}(u)\right)^{\frac{1}{r}} \leq C\|\nabla u\|_{L^{p}(\Omega)} .
$$

Proof. Suppose that (2.8) is not true. Then we can find a sequence $\left(u_{n}\right)_{n} \subset \mathcal{C}_{r} \subset W$ such that $\mathcal{K}_{r}\left(u_{n}\right)=1$ and

$$
\left\|\nabla u_{n}\right\|_{L^{p}(\Omega)} \leq \frac{1}{n} \forall n \geq 1
$$


Clearly, from Lemma 2.1 and (2.9), the sequence $\left(u_{n}\right)_{n}$ is bounded in $W$, thus, by passing to a subsequence if necessary, we may assume that there exists $u_{0} \in W$ such that $u_{n} \rightarrow u$ as $n \rightarrow \infty$. Since $W$ is embedded compactly in $L^{r}(\Omega)$ and $L^{r}(\partial \Omega)$ we have that

$$
u_{n} \rightarrow u_{0} \text { in } L^{r}(\Omega), u_{n} \rightarrow u_{0} \text { in } L^{r}(\partial \Omega) .
$$

As $\mathcal{K}_{r}\left(u_{n}\right)=1 \forall n \geq 1$ and $\left(u_{n}\right)_{n} \subset \mathcal{C}_{r}$ we have $\mathcal{K}_{r}\left(u_{0}\right)=1$ and $u_{0} \in \mathcal{C}_{r}$. On the other hand, from (2.9), the sequence $\left(\left\|\nabla\left(u_{n}\right)\right\|_{L^{p}(\Omega)}\right)_{n}$ tends to 0 . Therefore $\nabla\left(u_{0}\right) \equiv 0$, so $u_{0}$ is constant and belongs to $\mathcal{C}_{r}$, hence $u_{0} \equiv 0$. This contradicts the fact that $\mathcal{K}_{r}\left(u_{0}\right)=1$.

\section{Proof of Theorem 1.1}

We have already stated that $\lambda_{0}=0$ is an eigenvalue of problem (1.1) and any other eigenvalue of this problem belongs to $(0, \infty)$.

In what follows we fix $\lambda>0$ and define $\mathcal{J}_{\lambda}: W \rightarrow \mathbb{R}$,

$$
\mathcal{J}_{\lambda}(u)=\frac{1}{p} \int_{\Omega}|\nabla u|^{p} d x+\frac{1}{q} \int_{\Omega}|\nabla u|^{q} d x-\frac{\lambda}{r} \mathcal{K}_{r}(u),
$$

which is a $C^{1}$ functional whose derivative is given by

$$
\begin{aligned}
\left\langle\mathcal{J}_{\lambda}^{\prime}(u), w\right\rangle & =\int_{\Omega}|\nabla u|^{p-2} \nabla u \cdot \nabla w d x+\int_{\Omega}|\nabla u|^{q-2} \nabla u \cdot \nabla w d x \\
& -\lambda\left(\int_{\Omega} a|u|^{r-2} u w d x+\int_{\partial \Omega} b|u|^{r-2} u w d \sigma\right) \forall u, w \in W .
\end{aligned}
$$

So, according to Definition 1.1, $\lambda>0$ is an eigenvalue of problem (1.1) if and only if there exists a critical point $u_{\lambda} \in W \backslash\{0\}$ of $\mathcal{J}_{\lambda}$, i. e. $\mathcal{J}_{\lambda}^{\prime}\left(u_{\lambda}\right)=0$.

The proof of Theorem 1.1 will follow as a consequence of several intermediate results.

We shall discuss two distinct cases.

Case 1: $\left(h_{p q r}\right)$ with $1<r<q<p<\infty$ and $\left(h_{a b}\right)$

The following result shows that, for every $\lambda>0$, the functional defined in (3.11), restricted to the subset $\mathcal{C}_{r} \subset W$, is coercive.

Lemma 3.3. If hypotheses ( $\left.h_{p q r}\right)$ with $1<r<p<\infty$ and $\left(h_{a b}\right)$ hold, then for every $\lambda>0$, we have $\lim _{\|u\|_{W} \rightarrow \infty, u \in \mathcal{C}_{r}} \mathcal{J}_{\lambda}(u)=\infty$.

Proof. We know from Lemma 2.2 (for $p=r$ ) that there exists a positive constant $C$ such that (2.8) holds. Using Hölder's inequality we have,

$$
\mathcal{K}_{r}(u) \leq C^{r}\|\nabla u\|_{L^{r}(\Omega)}^{r} \leq C^{r}|\Omega|_{N}^{\frac{p-r}{p}}\|\nabla u\|_{L^{p}(\Omega)}^{\frac{r}{p}} \forall u \in \mathcal{C}_{r} .
$$

Here by $|\cdot|_{N}$ we denote the Lebesgue measure on $\mathbb{R}^{N}$. So, we obtain from (3.12) that

$$
\mathcal{J}_{\lambda}(u) \geq \frac{1}{p}\|\nabla u\|_{L^{p}(\Omega)}^{p}-\frac{\lambda}{r} C^{r}|\Omega|_{N}^{\frac{p-r}{p}}\|\nabla u\|_{L^{p}(\Omega)}^{\frac{r}{p}} \quad \forall u \in \mathcal{C}_{r} .
$$

Taking into account Lemma 2.1, Lemma 2.2 and (3.12), we can see that $\|u\|_{W} \rightarrow \infty, u \in \mathcal{C}_{r}$ if and only if $\|\nabla u\|_{L^{p}(\Omega)} \rightarrow \infty$. Since $r<p$, we derive from (3.13) that $\mathcal{J}_{\lambda}(u) \rightarrow \infty$ if $\|u\|_{W} \rightarrow \infty, u \in \mathcal{C}_{r}$, therefore $\mathcal{J}_{\lambda}$ is indeed coercive on $\mathcal{C}_{r}$.

Proposition 3.1. In Case 1, every number $\lambda>0$ is an eigenvalue of problem (1.1).

Proof. Note that $\mathcal{C}_{r}$ is a weakly closed subset of the reflexive Banach space $W$, and functional $\mathcal{J}_{\lambda}$ is coercive (see Lemma 3.3) and weakly lower semicontinuous on $\mathcal{C}_{r}$ with respect to the norm of $W$. Standard results in the calculus of variations (see, e.g., [13, Theorem 1.2]) ensures the existence of a global minimizer $u_{*} \in \mathcal{C}_{r}$ for $\mathcal{J}_{\lambda}$, i.e., $\mathcal{J}_{\lambda}\left(u_{*}\right)=\min _{\mathcal{C}_{r}} \mathcal{J}_{\lambda}$. 
Next, we are going to prove that $u_{*} \not \equiv 0$.

Let us choose $u_{0} \in \mathcal{C}_{r} \backslash\{0\}$ such that $\mathcal{K}_{r}\left(u_{0}\right)>0$ (see [4, Section 2] for the construction of such a function). Note that the function

$$
t \mapsto \mathcal{J}_{\lambda}\left(t u_{0}\right)=t^{r}\left(\frac{t^{p-r}}{p} \int_{\Omega}\left|\nabla u_{0}\right|^{p} d x+\frac{t^{q-r}}{q} \int_{\Omega}\left|\nabla u_{0}\right|^{q} d x-\frac{\lambda}{r} \mathcal{K}_{r}\left(u_{0}\right)\right),
$$

is negative for $t=t_{0}>0$ small enough. Therefore, as $t u_{0} \in \mathcal{C}_{r} \backslash\{0\}$, we have $\mathcal{J}_{\lambda}\left(u_{*}\right)<0$, so $u_{*} \not \equiv 0$.

Next, we are going to show that the global minimizer $u_{*}$ for $\mathcal{J}_{\lambda}$ restricted to $\mathcal{C}_{r}$ is a critical point of $\mathcal{J}_{\lambda}$ considered on the whole space $W$, i. e., $\mathcal{J}_{\lambda}^{\prime}\left(u_{*}\right)=0$, in other words, $u_{*}$ is an eigenfunction of problem (1.1) corresponding to $\lambda$.

In order to show this we make use of an argument similar to that used in [5] and [3, Lemma 3]. In this respect, we fix $v \in \operatorname{Lip}(\Omega)$ arbitrarily. For each $n \in \mathbb{N}^{*}$ define $f_{n}: \mathbb{R} \rightarrow \mathbb{R}$,

$$
f_{n}(s):=\mathcal{K}_{r}\left(u_{*}+\frac{1}{n} v+s\right)=\int_{\Omega} a\left|u_{*}+\frac{1}{n} v+s\right|^{r} d x+\int_{\partial \Omega} b\left|u_{*}+\frac{1}{n} v+s\right|^{r} d \sigma .
$$

It is easily seen that $f_{n}$ is coercive, since we have

$$
f_{n}(s) \geq \frac{|s|^{r}}{2^{r}}\left(\int_{\Omega} a d x+\int_{\partial \Omega} b d \sigma\right)-\int_{\Omega} a\left|u_{*}+\frac{1}{n} v\right|^{r} d x-\int_{\partial \Omega} b\left|u_{*}+\frac{1}{n} v\right|^{r} d \sigma .
$$

We have used the inequality

$$
|x|^{r} \leq(|x+y|+|y|)^{r} \leq 2^{r}\left(|x+y|^{r}+|y|^{r}\right) \forall x, y \in \mathbb{R}, r>1 .
$$

Moreover, function $f_{n}$ is continuously differentiable on $\mathbb{R}$ (see [10, Theorem 2.27]) and convex (its derivative is an increasing function). Therefore, for all $n \in \mathbb{N}^{*}, f_{n}$ admits a minimum point $s_{n}$, such that $f_{n}^{\prime}\left(s_{n}\right)=0$, that is

$$
\begin{aligned}
\int_{\Omega} a \mid u_{*} & +\frac{1}{n} v+\left.s_{n}\right|^{r-2}\left(u_{*}+\frac{1}{n} v+s_{n}\right) d x \\
& +\int_{\partial \Omega} b\left|u_{*}+\frac{1}{n} v+s_{n}\right|^{r-2}\left(u_{*}+\frac{1}{n} v+s_{n}\right) d \sigma=0
\end{aligned}
$$

We denote

$$
u_{n}:=u_{*}+\frac{1}{n} v+s_{n} \forall n \in \mathbb{N}^{*} .
$$

According to (3.14), $\left(u_{n}\right)_{n} \subset \mathcal{C}_{r}$.

Next, we claim that the sequence $\left(n s_{n}\right)_{n}$ is bounded. Arguing by contradiction, let us assume that, up to a sequence, $n s_{n} \rightarrow \infty$ or $n s_{n} \rightarrow-\infty$ as $n \rightarrow \infty$. Taking into account that $v \in \operatorname{Lip}(\Omega)$ there exists $N_{1}$ large enough such that we have either

$$
v(\cdot)+n s_{n}>0 \text { in } \Omega \text {, or } v(\cdot)+n s_{n}<0 \text { in } \Omega \forall n \geq N_{1} \text {. }
$$

Since the function $\tau \mapsto\left|u_{*}+\tau\right|^{r-2}\left(u_{*}+\tau\right)$ is strictly increasing on $\mathbb{R}$, we get

$$
\begin{aligned}
0 & =\int_{\Omega} a\left|u_{n}\right|^{r-2} u_{n} d x+\int_{\partial \Omega} b\left|u_{n}\right|^{r-2} u_{n} d \sigma \\
& >\int_{\Omega} a\left|u_{*}\right|^{r-2} u_{*} d x+\int_{\partial \Omega} b\left|u_{*}\right|^{r-2} u_{*} d \sigma=0 \forall n \geq N_{1},
\end{aligned}
$$

if $v(\cdot)+n s_{n}>0$ in $\Omega$, or the reverse inequality in the later case, when $v(\cdot)+n s_{n}<0$ in $\Omega$. In both cases we get a contradiction.

We point out that the inequality in (3.16) is strict. Indeed, (1.2) implies that either $|\{x \in \Omega ; a(x)>0\}|_{N}>0$ or $a=0$ a.e. in $\Omega$ and $|\{x \in \partial \Omega ; b(x)>0\}|_{N-1}>0$, hence we can not have equality above, instead of " $>$ ". 
Consequently, $\left(n s_{n}\right)_{n}$ should be bounded. This implies that there exists $S \in \mathbb{R}$ such that, up to a subsequence, $n s_{n} \rightarrow S$ as $n \rightarrow \infty$. Therefore, on a subsequence, we have

$$
n\left(u_{n}-u_{*}\right) \rightarrow v+S \text { and } u_{n} \rightarrow u_{*} \text { in } W \text { as } n \rightarrow \infty \text {. }
$$

In addition, there exists $N_{2} \in \mathbb{N}^{*}$ such that $u_{n} \not \equiv 0 \forall n \geq N_{2}$. By using the minimality of $u_{*}$ and the fact that $u_{n} \in \mathcal{C}_{r} \backslash\{0\} \forall n \geq N_{2}$, we obtain that

$$
0 \leq \lim _{n \rightarrow \infty} \frac{\mathcal{J}_{\lambda}\left(u_{n}\right)-\mathcal{J}_{\lambda}\left(u_{*}\right)}{(1 / n)}
$$

On the other hand,

$$
n\left(\mathcal{J}_{\lambda}\left(u_{n}\right)-\mathcal{J}_{\lambda}\left(u_{*}\right)\right)=\left\langle\mathcal{J}_{\lambda}^{\prime}\left(u_{*}\right), n\left(u_{n}-u_{*}\right)\right\rangle+o\left(n ; u_{*}, v\right),
$$

where $o\left(n ; u_{*}, v\right)$ is a notation for the term which tends to zero in the definition of the Fréchet derivative of $\mathcal{J}_{\lambda}$ at $u_{*}$, that is $o\left(n ; u_{*}, v\right) \rightarrow 0$ as $n \rightarrow \infty$. It follows from (3.17)(3.19) in combination with $u_{*} \in \mathcal{C}_{r}$ that

$$
\begin{aligned}
0 \leq & \lim _{n \rightarrow \infty} n\left(\mathcal{J}_{\lambda}\left(u_{n}\right)-\mathcal{J}_{\lambda}\left(u_{*}\right)\right)=\lim _{n \rightarrow \infty}\left\langle\mathcal{J}_{\lambda}^{\prime}\left(u_{*}\right), n\left(u_{n}-u_{*}\right)\right\rangle+o\left(n ; u_{*}, v\right) \\
& =\left\langle\mathcal{J}_{\lambda}^{\prime}\left(u_{*}\right), v+S\right\rangle=\left\langle\mathcal{J}_{\lambda}^{\prime}\left(u_{*}\right), v\right\rangle .
\end{aligned}
$$

A similar reasoning with $-v$ instead of $v$ and the density of Lipschitz functions in $W$ yield $\mathcal{J}_{\lambda}^{\prime}\left(u_{*}\right)=0$, which concludes the proof.

Case 2: $\left(h_{p q r}\right)$ with $1<q<p<r<\infty$ and $\left(h_{a b}\right)$

Let $\lambda>0$ be a fixed number. Under the assumption $1<q<p<r<\infty$ we cannot expect coercivity of the functional $\mathcal{J}_{\lambda}$ on $\mathcal{C}_{r}$. From now on we analyse the action of $\mathcal{J}_{\lambda}$ on the Nehari type manifold (see [14]) defined by

$$
\begin{aligned}
\mathcal{N}_{\lambda} & =\left\{v \in \mathcal{C}_{r} \backslash\{0\} ;\left\langle\mathcal{J}_{\lambda}^{\prime}(w), w\right\rangle=0\right\} \\
& =\left\{w \in \mathcal{C}_{r} \backslash\{0\} ; \int_{\Omega}\left(|\nabla w|^{p}+|\nabla w|^{q}\right) d x=\lambda \mathcal{K}_{r}(w)\right\} .
\end{aligned}
$$

It is natural to consider the restriction of $\mathcal{J}_{\lambda}$ to $\mathcal{N}_{\lambda}$ since any possible eigenfunction corresponding to $\lambda$ belongs to $\mathcal{N}_{\lambda}$. Note that on $\mathcal{N}_{\lambda}$ functional $\mathcal{J}_{\lambda}$ has the form

$$
\mathcal{J}_{\lambda}(u)=\frac{r-p}{p r} \int_{\Omega}|\nabla u|^{p} d x+\frac{r-q}{q r} \int_{\Omega}|\nabla u|^{p} d x>0
$$

(see also Remark 2.3).

We have

Lemma 3.4. In Case 2, for every $\lambda>0$ we have $\mathcal{N}_{\lambda} \neq \emptyset$.

Proof. We fix $u_{0} \in \mathcal{C}_{r} \backslash\{0\}$ such that $\mathcal{K}_{r}\left(u_{0}\right)>0$. We claim that for a convenient $t>0$, $t u_{0} \in \mathcal{N}_{\lambda}$. Since $\mathcal{C}_{r}$ is a cone, $t u_{0} \in \mathcal{C}_{r}$ for all $t \in \mathbb{R}$. So the condition $t u_{0} \in \mathcal{N}_{\lambda}, t>0$, reads

$$
h(t):=t^{p} \int_{\Omega}\left|\nabla u_{0}\right|^{p} d x+t^{q} \int_{\Omega}\left|\nabla u_{0}\right|^{q} d x-\lambda t^{r} \mathcal{K}_{r}\left(u_{0}\right)=0 .
$$

Noting that the function $t \mapsto h(t)$ is continuous on $(0, \infty)$ and

$$
\begin{gathered}
t^{-q} h(t) \rightarrow \int_{\Omega}\left|\nabla u_{0}\right|^{q} d x>0 \text { as } t \rightarrow 0^{+}, \\
t^{-r} h(t) \rightarrow-\lambda \mathcal{K}_{r}\left(u_{0}\right)<0 \text { as } t \rightarrow \infty,
\end{gathered}
$$

we infer that there exists $t_{0} \in(0, \infty)$ such that $h\left(t_{0}\right)=0$, so $t_{0} u_{0} \in \mathcal{N}_{\lambda}$. 
Lemma 3.5. If hypotheses $\left(h_{p q r}\right)$ with $1<q<p<r<\infty$ and $\left(h_{a b}\right)$ hold, then there exists a point $u_{*} \in \mathcal{N}_{\lambda}$ where $\mathcal{J}_{\lambda}$ attains its minimal value,

$$
m_{\lambda}:=\inf _{w \in \mathcal{N}_{\lambda}} \mathcal{J}_{\lambda}(w)>0 .
$$

Proof. Let $\left(u_{n}\right)_{n} \subset \mathcal{N}_{\lambda}$ be a minimizing sequence for $\mathcal{J}_{\lambda}$. Since $u_{n} \in \mathcal{N}_{\lambda}$ for all $n$, we obtain from (3.21)

$$
\mathcal{J}_{\lambda}\left(u_{n}\right)=\frac{r-p}{p r} \int_{\Omega}\left|\nabla u_{n}\right|^{p} d x+\frac{r-q}{q r} \int_{\Omega}\left|\nabla u_{n}\right|^{q} d x \rightarrow m_{\lambda} \geq 0, \text { as } n \rightarrow \infty .
$$

On the other hand, we have

$$
\int_{\Omega}\left|\nabla u_{n}\right|^{p} d x+\int_{\Omega}\left|\nabla u_{n}\right|^{q} d x=\lambda \mathcal{K}_{r}\left(u_{n}\right) \forall n \geq 1 .
$$

Now, from (3.22) we obtain that $\left(\left\|\nabla u_{n}\right\|_{L^{p}(\Omega)}\right)_{n}$ and $\left(\left\|\nabla u_{n}\right\|_{L^{q}(\Omega)}\right)_{n}$ are bounded sequences, therefore taking into account (3.23), we can see that $\left(\mathcal{K}_{r}\left(u_{n}\right)\right)_{n}$ is also a bounded sequence and making use of Lemma 2.1 we obtain that $\left(u_{n}\right)_{n}$ is bounded in $W$.

Next, let us prove that $m_{\lambda}=\inf _{w \in \mathcal{N}_{\lambda}} \mathcal{J}_{\lambda}(w)>0$. Assume that, on the contrary, $m_{\lambda}=0$. Let $\left(u_{n}\right)_{n} \subset \mathcal{N}_{\lambda}$ be a minimizing sequence for $\mathcal{J}_{\lambda}$. Note that $\mathcal{K}_{r}\left(u_{n}\right)>0$ for all $n$ (see Remark 2.3). We have (see (3.22))

$$
\mathcal{J}_{\lambda}\left(u_{n}\right)=\frac{r-p}{p r} \int_{\Omega}\left|\nabla u_{n}\right|^{p} d x+\frac{r-q}{q r} \int_{\Omega}\left|\nabla u_{n}\right|^{q} d x \rightarrow 0 \text { as } n \rightarrow \infty .
$$

We know that $\left(u_{n}\right)_{n}$ is bounded in $W$, so there exists $u_{0} \in W$ such that, on a subsequence denoted again $\left(u_{n}\right)_{n}, u_{n} \rightarrow u_{0}$ in $W$ (hence also in $W^{1, q}(\Omega)$ ), and $u_{n} \rightarrow u_{0}$ in $L^{r}(\Omega)$, $u_{n} \rightarrow u_{0}$ in $L^{r}(\partial \Omega)$. Clearly $u_{0} \in \mathcal{C}_{r}$ and from (3.24) we deduce that $u_{0}$ is a constant function, so $u_{0} \equiv 0$. Summarizing, we have proved that

$$
u_{n} \rightarrow 0 \text { in } W, \mathcal{K}_{r}\left(u_{n}\right) \rightarrow 0 \text { as } n \rightarrow \infty .
$$

We define $v_{n}:=u_{n} /\left(\mathcal{K}_{r}\left(u_{n}\right)\right)^{1 / r}, n \geq 1$. By (3.23) we can see that

$$
0<\int_{\Omega}\left|\nabla u_{n}\right|^{q} d x=\lambda \mathcal{K}_{r}\left(u_{n}\right)-\int_{\Omega}\left|\nabla u_{n}\right|^{p} d x \forall n \geq 1 .
$$

Dividing this inequality by $\left(\mathcal{K}_{r}\left(u_{n}\right)\right)^{p / r}$, we obtain

$$
\int_{\Omega}\left|\nabla v_{n}\right|^{p} d x<\lambda\left(\mathcal{K}_{r}\left(u_{n}\right)\right)^{\frac{r-p}{r}} \forall n \geq 1 .
$$

Since $p<r$ and $\left(\mathcal{K}_{r}\left(u_{n}\right)\right)^{\frac{r-p}{r}} \rightarrow 0$ as $n \rightarrow \infty$, we get by (3.26) that $\left(\left\|\nabla v_{n}\right\|_{L^{p}(\Omega)}\right)_{n}$ is a bounded sequence. In addition, $\mathcal{K}_{r}\left(v_{n}\right)=1$ for all $n \geq 1$, thus, $\left(v_{n}\right)_{n}$ is bounded in $W$ (see Lemma 2.1). As $\left(v_{n}\right)_{n}$ is a sequence in $\mathcal{C}_{r}$ which is weakly closed in $W$, it follows that there exists a $v_{0} \in \mathcal{C}_{r}$ such that, on a subsequence, $v_{n} \rightarrow v_{0}$ in $W$ and $v_{n} \rightarrow v_{0}$ in $L^{r}(\Omega)$ as well as in $L^{r}(\partial \Omega)$.

Next, since $v_{n} \rightarrow v_{0}$ in $W$, we infer from (3.26)

$$
\int_{\Omega}\left|\nabla v_{0}\right|^{p} d x \leq \liminf _{n \rightarrow \infty} \int_{\Omega}\left|\nabla v_{n}\right|^{p} d x=0
$$

Therefore $v_{0}$ is a constant function and in fact $v_{0} \equiv 0$ since $v_{0} \in \mathcal{C}_{r}$. Thus, $v_{n} \rightarrow 0$ in both $L^{r}(\Omega)$ and $L^{r}(\partial \Omega)$. But this contradicts the fact that $\mathcal{K}_{r}\left(v_{n}\right)=1 \forall n \geq 1$. This contradiction shows that $m_{\lambda}>0$.

Finally, we are going to prove that there exists $u_{*} \in \mathcal{N}_{\lambda}$ such that $\mathcal{J}_{\lambda}\left(u_{*}\right)=m_{\lambda}$. 
Let $\left(u_{n}\right)_{n} \subset \mathcal{N}_{\lambda}$ be a minimizing sequence: $\mathcal{J}_{\lambda}\left(u_{n}\right) \rightarrow m_{\lambda}$. Since $\left(u_{n}\right)_{n}$ is bounded in $W$, on a subsequence, $\left(u_{n}\right)_{n}$ converges weakly in $W$ to some $u_{*} \in W$ and strongly in both $L^{r}(\Omega)$ and $L^{r}(\partial \Omega)$ (to the same $u_{*}$ ). Thus,

$$
\mathcal{J}_{\lambda}\left(u_{*}\right) \leq \liminf _{n \rightarrow \infty} \mathcal{J}_{\lambda}\left(u_{n}\right)=m_{\lambda}
$$

As $\left(u_{n}\right)_{n} \subset \mathcal{N}_{\lambda}$ we have

$$
\int_{\Omega}\left(\left|\nabla u_{n}\right|^{p}+\left|\nabla u_{n}\right|^{q}\right) d x=\lambda \mathcal{K}_{r}\left(u_{n}\right) \forall n \geq 1 .
$$

It is easily seen that $u_{*}$ is not the null function. Indeed, assuming that $u_{*} \equiv 0$, we infer by (3.28) that $\left(u_{n}\right)_{n}$ converges strongly to 0 in $W$ and $\left\|\nabla u_{n}\right\|_{L^{q}(\Omega)}^{q} \rightarrow 0$ as $n \rightarrow \infty$. Then (3.22) will give $m_{\lambda}=0$ which is a contradiction. Obviously $u_{*} \in \mathcal{C}_{r} \backslash\{0\}$. Letting $n \rightarrow \infty$ in (3.28) yields

$$
\int_{\Omega}\left(\left|\nabla u_{*}\right|^{p}+\left|\nabla u_{*}\right|^{q}\right) d x \leq \lambda \mathcal{K}_{r}\left(u_{*}\right) .
$$

If (3.29) holds with equality then we are done. We shall prove that assuming strict inequality in (3.29) leads to a contradiction. Thus, let us assume that

$$
\int_{\Omega}\left(\left|\nabla u_{*}\right|^{p}+\left|\nabla u_{*}\right|^{q}\right) d x<\lambda \mathcal{K}_{r}\left(u_{*}\right) .
$$

Now, we can choose $t_{0} \in(0,1)$ such that $t_{0} u_{*} \in \mathcal{N}_{\lambda}$. Indeed if we define $j:(0, \infty) \rightarrow \mathbb{R}$,

$$
j(t):=t^{r}\left(\int_{\Omega}\left(t^{p-r}\left|\nabla u_{*}\right|^{p}+t^{q-r}\left|\nabla u_{*}\right|^{q}\right) d x-\lambda \mathcal{K}_{r}\left(u_{*}\right)\right)
$$

we have $j(1)<0$ (see (3.30)) and $t^{-r} j(t) \rightarrow \infty$ as $t \rightarrow 0_{+}$. Therefore, there exists $t_{0} \in$ $\left(\delta_{0}, 1\right)$ such that $j\left(t_{0}\right)=0$, which implies $t_{0} u_{*} \in \mathcal{N}_{\lambda}$.

Next, using the form of $\mathcal{J}_{\lambda}$ on the Nehari manifold $\mathcal{N}_{\lambda}$, we get

$$
\mathcal{J}_{\lambda}\left(t_{0} u_{*}\right)=\frac{t_{0}^{p}(r-p)}{p r} \int_{\Omega}\left|\nabla u_{*}\right|^{p} d x+\frac{t_{0}^{q}(r-q)}{q r} \int_{\Omega}\left|\nabla u_{*}\right|^{q} d x .
$$

Therefore,

$$
\begin{aligned}
0< & m_{\lambda} \leq \mathcal{J}_{\lambda}\left(t_{0} u_{*}\right)=\frac{t_{0}^{p}(r-p)}{p r} \int_{\Omega}\left|\nabla u_{*}\right|^{p} d x+\frac{t_{0}^{q}(r-q)}{q r} \int_{\Omega}\left|\nabla u_{*}\right|^{q} d x \\
& <t_{0}^{q}\left(\frac{r-p}{p r} \int_{\Omega}\left|\nabla u_{*}\right|^{p} d x+\frac{r-q}{q r} \int_{\Omega}\left|\nabla u_{*}\right|^{q} d x\right) \\
& \leq \frac{r-p}{p r} \liminf _{n \rightarrow \infty}\left(\int_{\Omega}\left|\nabla u_{n}\right|^{p} d x\right)+\frac{r-q}{q r} \liminf _{n \rightarrow \infty}\left(\int_{\Omega}\left|\nabla u_{n}\right|^{q} d x\right) \\
& \leq \liminf _{n \rightarrow \infty} \mathcal{J}_{\lambda}\left(u_{n}\right)=m_{\lambda},
\end{aligned}
$$

which is impossible.

The next result states that the minimizer $u_{*}$, given by Lemma 3.5, is a critical point of $\mathcal{J}_{\lambda}$ considered on the whole space $W$.

Proposition 3.2. In Case 2, the minimizer $u_{*} \in \mathcal{N}_{\lambda}$ from Lemma 3.5 is an eigenfunction of problem (1.1) with corresponding eigenvalue $\lambda$. 
Proof. It suffices to prove that $\mathcal{J}_{\lambda}^{\prime}\left(u_{*}\right)=0$. So, let $v \in \operatorname{Lip}(\Omega)$ be an arbitrary but fixed function and let $u_{*} \in \mathcal{N}_{\lambda}$ be the minimizer of $\mathcal{J}_{\lambda}$ over $\mathcal{N}_{\lambda}$. As in the Case 1 (see Proposition 3.1) we are able to obtain a sequence $\left(u_{n}\right)_{n} \subset \mathcal{C}_{r} \backslash\{0\}$,

$$
u_{n}:=u_{*}+\frac{1}{n} v+s_{n} \forall n \geq 1 .
$$

The sequence $\left(n s_{n}\right)_{n}$ is also bounded, so it converges, on a subsequence, to some $S \in \mathbb{R}$. Therefore, we have

$$
n\left(u_{n}-u_{*}\right) \rightarrow v+S, u_{n} \rightarrow u_{*} \text { in } W \text { as } n \rightarrow \infty .
$$

Since $u_{*} \in \mathcal{K}_{r}\left(u_{*}\right)$ and $\mathcal{K}_{r}\left(u_{n}\right) \rightarrow \mathcal{K}_{r}\left(u_{*}\right)>0$, one can assume that $\mathcal{K}_{r}\left(u_{n}\right)>0$ for all $n \geq 1$.

Using the sequence $\left(u_{n}\right)_{n^{\prime}}$ we shall construct a sequence $\left(t_{n}\right)_{n} \subset \mathbb{R} \backslash\{0\}$ such that, up to a subsequence, $\left(t_{n} u_{n}\right)_{n} \subset \mathcal{N}_{\lambda}$, i.e.,

$$
t_{n}^{p-r} \int_{\Omega}\left|\nabla u_{n}\right|^{p} d x+t_{n}^{q-r} \int_{\Omega}\left|\nabla u_{n}\right|^{q} d x=\lambda \mathcal{K}_{r}\left(u_{n}\right)
$$

Let us show that for every $n \geq 1$ there exists $t_{n}>0$ such that (3.34) holds. Define

$$
h_{n}:(0, \infty) \rightarrow \mathbb{R}, h_{n}(t):=t^{p-r} \int_{\Omega}\left|\nabla u_{n}\right|^{p} d x+t^{q-r} \int_{\Omega}\left|\nabla u_{n}\right|^{q} d x-\lambda \mathcal{K}_{r}\left(u_{n}\right) .
$$

Obviously, $h_{n}(t) \rightarrow \infty$ as $t \rightarrow 0+$ and $h_{n}(t) \rightarrow-\lambda \mathcal{K}_{a b}\left(u_{n}\right)<0$ as $t \rightarrow \infty$. So, there exists $t_{n}>0$ such that $h_{n}\left(t_{n}\right)=0 \forall n \geq 1$, hence (3.34) holds, as claimed.

In what follows we shall prove that the sequence $\left(n\left(t_{n}-1\right)\right)_{n}$ is bounded. To this purpose, we rewrite (3.34) in the equivalent form

$$
n\left(t_{n}^{p-r}-1\right) A\left(u_{n}\right)+n\left(t_{n}^{q-r}-1\right) B\left(u_{n}\right)=n\left(\lambda \mathcal{K}_{r}\left(u_{n}\right)-A\left(u_{n}\right)-B\left(u_{n}\right)\right),
$$

where $A\left(u_{n}\right):=\int_{\Omega}\left|\nabla u_{n}\right|^{p} d x, B\left(u_{n}\right):=\int_{\Omega}\left|\nabla u_{n}\right|^{q} d x$.

We shall prove first that the sequence $\left(n\left(\lambda \mathcal{K}_{r}\left(u_{n}\right)-A\left(u_{n}\right)-B\left(u_{n}\right)\right)\right)_{n}$ is convergent. To this purpose, let us define the $C^{1}$-functional $\mathcal{L}_{\lambda}: W \rightarrow \mathbb{R}$,

$$
\mathcal{L}_{\lambda}(u)=-\int_{\Omega}|\nabla u|^{p} d x-\int_{\Omega}|\nabla u|^{q} d x+\lambda \mathcal{K}_{r}(u) \forall u \in W .
$$

For all $u, w \in W$

$$
\begin{aligned}
\left\langle\mathcal{L}_{\lambda}^{\prime}(u), w\right\rangle & =-p \int_{\Omega}|\nabla u|^{p-2} \nabla u \cdot \nabla w d x-q \int_{\Omega}|\nabla u|^{q-2} \nabla u \cdot \nabla w d x \\
& +\lambda r\left(\int_{\Omega} a|u|^{r-2} u w d x+\int_{\partial \Omega} b\left|u_{\lambda}\right|^{r-2} u w d \sigma\right) .
\end{aligned}
$$

From (3.36) and $u_{*} \in \mathcal{N}_{\lambda}$, we infer that $\mathcal{L}_{\lambda}\left(u_{*}\right)=0$, so we get

$$
n\left(\lambda \mathcal{K}_{r}\left(u_{n}\right)-A\left(u_{n}\right)-B\left(u_{n}\right)\right)=n\left(\mathcal{L}_{\lambda}\left(u_{n}\right)-\mathcal{L}_{\lambda}\left(u_{*}\right)\right) .
$$

We have

$$
n\left(\mathcal{L}_{\lambda}\left(u_{n}\right)-\mathcal{L}_{\lambda}\left(u_{*}\right)\right) \rightarrow\left\langle\mathcal{L}_{\lambda}^{\prime}\left(u_{*}\right), v+S\right\rangle \text { as } n \rightarrow \infty .
$$

From (3.38) and (3.39) we deduce that the sequence $\left(n\left(\lambda \mathcal{K}_{r}\left(u_{n}\right)-A\left(u_{n}\right)-B\left(u_{n}\right)\right)\right.$ has a finite limit.

Returning to (3.35), we observe that $\left(A\left(u_{n}\right)\right)_{n},\left(B\left(u_{n}\right)\right)_{n}$ are bounded sequences of positive numbers. If we assume the contrary, that the sequence $\left(n\left(t_{n}^{p-r}-1\right)\right)_{n}$ has an unbounded subsequence converging, e.g., to $+\infty$, then the corresponding subsequence of $\left(n\left(t_{n}^{q-r}-1\right)\right)_{n}$ will have positive terms (since $q-r<0$ and $p-r<0$ ), so the sequence defined by the left hand side of (3.35) will be unbounded, thus contradicting the fact that 
the right hand side defines a convergent sequence. An analogue reasoning works in the case of a subsequence converging to $-\infty$. Therefore, $\left(n\left(t_{n}^{p-r}-1\right)\right)_{n}$ is a bounded sequence. Hence, there is $K>0$ such that for all $n \geq 1, n\left|t_{n}^{p-r}-1\right| \leq K$, which implies

$$
1-\frac{K}{n} \leq t_{n}^{p-r} \leq 1+\frac{K}{n} \forall n \geq 1 .
$$

Since, there exists $N_{1} \in \mathbb{N}^{*}$ such that $1-K / n>0 \forall n \geq N_{1}$, we have

$$
n\left(\left(1+\frac{K}{n}\right)^{\frac{1}{p-r}}-1\right) \leq n\left(t_{n}-1\right) \leq n\left(\left(1-\frac{K}{n}\right)^{\frac{1}{p-r}}-1\right) \forall n \geq N_{1} .
$$

Taking into account the relations

$$
\lim _{x \rightarrow 0} \frac{(1+K x)^{1 /(p-r)}-1}{x}=K /(p-r), \lim _{x \rightarrow 0} \frac{(1-K x)^{1 /(p-r)}-1}{x}=-K /(p-r),
$$

we infer from (3.40) that the sequence $\left(n\left(t_{n}-1\right)\right)_{n}$ is bounded, thus, by possibly passing to a subsequence, there exists $T \in \mathbb{R}$, such that $n\left(t_{n}-1\right) \rightarrow T$ as $n \rightarrow \infty$. We define

$$
z_{n}:=t_{n}\left(u_{*}+\frac{1}{n} v+s_{n}\right)=t_{n} u_{n} \forall n \geq N_{1},
$$

with $\left(z_{n}\right)_{n} \subset \mathcal{N}_{\lambda}$. In addition, as $\left(n\left(t_{n}-1\right)\right)_{n}$ is a bounded sequence, we can see that

$$
t_{n} \rightarrow 1 \text { in } \mathbb{R}, z_{n} \rightarrow u_{*} \text { in } W \text { as } n \rightarrow \infty .
$$

By using the minimality of $u_{*}$ and the fact that $\left(z_{n}\right)_{n} \subset \mathcal{N}_{\lambda}$ we obtain that

$$
0 \leq \lim _{n \rightarrow \infty} \frac{\mathcal{J}_{\lambda}\left(z_{n}\right)-\mathcal{J}_{\lambda}\left(u_{*}\right)}{\frac{1}{n}} .
$$

Since functional $\mathcal{J}_{\lambda} \in C^{1}(W ; \mathbb{R})$, we can write

$$
n\left(\mathcal{J}_{\lambda}\left(z_{n}\right)-\mathcal{J}_{\lambda}\left(u_{*}\right)\right)=\left(\left\langle\mathcal{J}_{\lambda}^{\prime}\left(u_{*}\right), n\left(z_{n}-u_{*}\right)\right\rangle+o\left(n ; u_{*}, v\right)\right.
$$

with $o\left(n ; u_{*}, v\right) \rightarrow 0$ as $n \rightarrow \infty$. Taking into account (3.41) and (3.42), we can see that

$$
n\left(z_{n}-u_{*}\right)=n\left(t_{n}-1\right) u_{*}+v+n s_{n} \rightarrow T u_{*}+v+S \text { as } n \rightarrow \infty \text { in } W .
$$

It follows from (3.43) and (3.45) that

$$
0 \leq\left\langle\mathcal{J}_{\lambda}^{\prime}\left(u_{*}\right), v+S+T u_{*}\right\rangle .
$$

Since $u_{*} \in \mathcal{N}_{\lambda}$, we obtain that $\left\langle\mathcal{J}_{\lambda}^{\prime}\left(u_{*}\right), u_{*}\right\rangle=0,\left\langle\mathcal{J}_{\lambda}^{\prime}\left(u_{*}\right), S\right\rangle=0$, hence (3.46) implies

$$
0 \leq\left\langle\mathcal{J}_{\lambda}^{\prime}\left(u_{*}\right), v\right\rangle \text {. }
$$

A similar reasoning with $-v$ instead of $v$ shows that the converse inequality holds, hence $0=\left\langle\mathcal{J}_{\lambda}^{\prime}\left(u_{*}\right), v\right\rangle$. Finally, using the density of Lipschitz functions in $W$ we obtain that $\mathcal{J}_{\lambda}^{\prime}\left(u_{*}\right)=0$, which concludes the proof.

Therefore, as it has already been pointed out, $\lambda=0$ is an eigenvalue, so the conclusion of Theorem 1.1 follows from Propositions 3.1 and 3.2.

Remark 3.4. As we have already mentioned in Introduction, in the case $p, q \in(1, \infty), p \neq$ $q, r \in\{p, q\}$, the set of eigenvalues of problem (1.1) has been completely determined in [3]. The case $1<q<r<p<\infty$ remains open. 


\section{REFERENCES}

[1] Abreu, J. and Madeira, G., Generalized eigenvalues of the $(p, 2)$-Laplacian under a parametric boundary condition, Proc. Edinburgh Math. Soc., 63 (2020), No. 1, 287-303

[2] Adams, R. A. and Fournier, J. J., Sobolev Spaces, second ed., Pure Appl. Math., 140, Academic Press, New York-London, 2003

[3] Barbu, L. and Moroşanu, G., Full description of the eigenvalue set of the ( $p, q)$-Laplacian with a Steklov-like boundary condition, J. Differential Equations, in press

[4] Barbu, L. and Moroşanu, G., Eigenvalues of the negative $(p, q)$-Laplacian under a Steklov-like boundary condition, Complex Var. Elliptic Equations, 64 (2019), No. 4, 685-700

[5] Brasco, L. and Franzina, G., An anisotropic eigenvalue problem of Stekloff type and weighted Wulff inequalities, Nonlinear Differ. Equ. Appl., 20 (2013), 1795-1830

[6] Brezis, H., Functional Analysis, Sobolev Spaces and Partial Differential Equations, Springer, 2011

[7] Casas, E. and Fernández, L. A., A Green's formula for quasilinear elliptic operators, J. Math. Anal. Appl., 142 (1989), 62-73

[8] Denkowski, Z., Migórski, S. and Papageorgiou, N. S., An Introduction to Nonlinear Analysis: Theory, Springer, New York, 2003

[9] Fărcăşeanu, M., Mihăilescu M. and Stancu-Dumitru, D., On the set of eigen-values of some PDEs with homogeneous Neumann boundary condition, Nonlinear Anal. Theory Methods Appl., 116 (2015), 19-25

[10] Folland, G. B., Real Analysis: Modern Techniques and Their Applications (2nd ed.), Pure and Applied Mathematics, John Wiley \& Sons, Inc., New York, 1999

[11] Mihăilescu, M., An eigenvalue problem possesing a continuous family of eigenvalues plus an isolated eigenvale, Commun. Pure Appl. Anal., 10 (2011), 701-708

[12] Mihăilescu, M. and Moroşanu, G., Eigenvalues of $-\triangle_{p}-\triangle_{q}$ under Neumann boundary condition, Canadian Math. Bull., 59 (2016), No. 3, 606-616

[13] Struwe, M., Variational Methods: Applications to Nonlinear Partial Differential Equations and Hamiltonian Systems, Springer, 1996

[14] Szulkin, A. and Weth, T., The Method of Nehari Manifold, Handbook of Nonconvex Analysis and Applications, Int. Press, Somerville, MA, 597-632, 2010

${ }^{a}$ OVIDIUS UNIVERSITY

FACUlty OF MATHEMATICS AND COMPUTER SCIENCE

DEPARTMENT OF MATHEMATICS AND COMPUTER SCIENCE

124 Mamaia Blvd, 900527, CONSTANŢA, ROMANiA

Email address: lbarbu@univ-ovidius.ro

${ }^{b}$ ACADEMY OF ROMANIAN SCIENTISTS, BUCHAREST, ROMANIA,

${ }^{c}$ BABEŞ-BOLYAI UNIVERSITY

FACULTY OF MATHEMATICS AND COMPUTER SCIENCE

1 M. KogĂLNiCEANU STR., 400084 Cluj-NAPOCA, ROMANIA

Email address: morosanu@math.ubbcluj.ro 